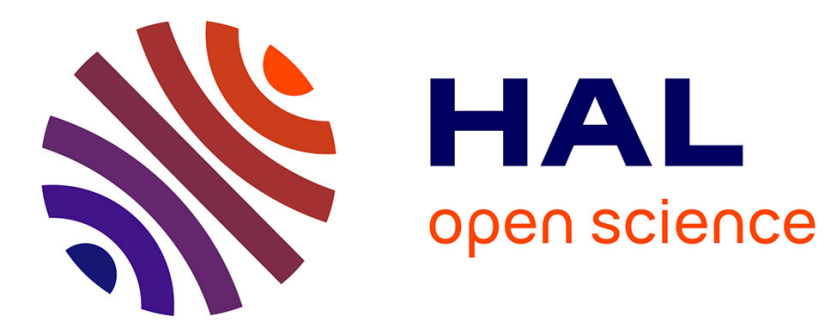

\title{
Turbulent spots and waves in a model for plane Poiseuille flow
}

\author{
Maher Lagha
}

\section{To cite this version:}

Maher Lagha. Turbulent spots and waves in a model for plane Poiseuille flow. Physics of Fluids, 2007, 19 (12), pp.124103. 10.1063/1.2821912 . hal-01023346

\section{HAL Id: hal-01023346 \\ https://hal-polytechnique.archives-ouvertes.fr/hal-01023346}

Submitted on $20 \mathrm{Jul} 2014$

HAL is a multi-disciplinary open access archive for the deposit and dissemination of scientific research documents, whether they are published or not. The documents may come from teaching and research institutions in France or abroad, or from public or private research centers.
L'archive ouverte pluridisciplinaire HAL, est destinée au dépôt et à la diffusion de documents scientifiques de niveau recherche, publiés ou non, émanant des établissements d'enseignement et de recherche français ou étrangers, des laboratoires publics ou privés. 


\section{AIP | Physics of Fluids}

\section{Turbulent spots and waves in a model for plane Poiseuille flow}

Maher Lagha

Citation: Physics of Fluids (1994-present) 19, 124103 (2007); doi: 10.1063/1.2821912

View online: http://dx.doi.org/10.1063/1.2821912

View Table of Contents: http://scitation.aip.org/content/aip/journal/pof2/19/12?ver=pdfcov

Published by the AIP Publishing

\section{Articles you may be interested in}

An apparent symmetry property of the mean velocity gradient in turbulent Poiseuille flows and its implications Phys. Fluids 23, 101705 (2011); 10.1063/1.3657819

Mean and turbulence structures of Couette-Poiseuille flows at different mean shear rates in a square duct Phys. Fluids 18, 068103 (2006); 10.1063/1.2210019

Investigation of simplified thermal expansion models for compressible Newtonian fluids applied to nonisothermal plane Couette and Poiseuille flows

Phys. Fluids 16, 3955 (2004); 10.1063/1.1787271

Influence of small imperfections on the stability of plane Poiseuille flow: A theoretical model and direct numerical simulation

Phys. Fluids 16, 2852 (2004); 10.1063/1.1760100

Experimental study of turbulent Poiseuille-Couette flow

Phys. Fluids 12, 865 (2000); 10.1063/1.870341

\section{A|P| Journal of}

Journal of Applied Physics is pleased to announce André Anders as its new Editor-in-Chief 


\title{
Turbulent spots and waves in a model for plane Poiseuille flow
}

\author{
Maher Lagha ${ }^{\text {a) }}$ \\ Laboratoire d'Hydrodynamique (LadHyX), École Polytechnique, F-91128 Palaiseau, France
}

(Received 21 June 2007; accepted 30 October 2007; published online 14 December 2007)

The structure of a turbulent spot in plane Poiseuille flow is investigated using a model derived from the Navier-Stokes equations through a Galerkin method. The mean profile of the streamwise velocity inside the turbulent spot has the characteristic flat profile of a turbulent Poiseuille flow. The waves developing at the wing tips of the spot have an asymmetric streamwise velocity with respect to the channel centerline, whereas their associated wall-normal velocity component is symmetric. On the outskirts of the spot, a large-scale flow occupying the full gap between the plates is observed. It is characterized by a streamwise inflow toward the spot and a spanwise outflow from the spot. A detailed comparison with the numerical simulations and the experiments in the literature shows that these results are in fair agreement with the main features of the transitional plane Poiseuille flow.

(C) 2007 American Institute of Physics. [DOI: 10.1063/1.2821912]

\section{INTRODUCTION}

Plane Poiseuille flow (pPf), shear flow between two fixed parallel plates driven by a pressure gradient, experiences a transition to turbulence marked by the nucleation and growth of turbulent spots, i.e., patches of turbulent flow scattered amidst laminar flow, when the Reynolds number $R$ (based on the half-channel height $h$ and the centerline velocity $U_{\mathrm{cl}}$ ) exceeds a certain threshold $R_{g}$ [e.g., $R_{g} \sim 1000$ for Carlson et al. (Ref. 1) and $R_{g} \sim 1100$ for Alavyoon et al. (Ref. 2)].

This kind of transition is not restricted to the pPf case but also occurs in other shear flows such as plane Couette flow $^{3}$ and boundary layer flows. ${ }^{4}$ Despite a large body of numerical $^{5-7}$ and laboratory ${ }^{1,3}$ experiments, many questions regarding such transition remain unanswered, such as the mechanisms involved in the growth of turbulent spots ${ }^{8,9}$ and in the self-sustainment of the turbulent state. ${ }^{10}$

In their experimental investigation of the transitional plane Poiseuille flow, Carlson et al. ${ }^{1}$ noted that the spot has a central turbulent area, in front of which there is a disturbed but not turbulent region, whereas at the wing tips of the spot there are oblique waves.

The origin of these waves was investigated by $\mathrm{Li}$ and Widnall, ${ }^{11}$ who modeled the spot by a moving patch of Reynolds stress. Their numerical simulations have shown that spatially damped oblique waves, resembling those observed at the front of a turbulent spot, are generated. Furthermore, the nature of these waves and their effect on the dynamics of the spot were studied numerically by Henningson et al. ${ }^{12}$ Due to the presence of the spot, the mean spanwise profile is inflectional, and oblique waves may grow and then break down into turbulence. However, the linear growth rate of these waves calculated by Henningson ${ }^{13}$ is too small compared to the observed one. Therefore, he suggested that the waves attain their large growth rate by some additional mechanisms. Moreover, the characteristics of these waves,

a)Electronic mail: maher@ladhyx.polytechnique.fr. measured by Henningson and Alfredsson ${ }^{14}$ using hot film anemometry, have been found to be in fair agreement with the theoretical Tollmien-Schlichting waves, i.e., the least stable mode of the Orr-Sommerfeld equation, as conjectured by Carlson et al. ${ }^{1}$

The role of these waves on the spreading of the spot was addressed by Alavyoon et al. ${ }^{2}$ By pointing out the absence of waves at the wing tips of the spots in the boundary layer flow, they concluded that the waves would be of no importance for the spreading itself, if the same spreading mechanism is at work in both plane Poiseuille and boundary layer flows.

Therefore, despite a large body of experiments and well described results, the dynamics of turbulent spots in pPf remains poorly understood and many questions, such as the nature of the observed waves and the mechanisms of maintenance of the turbulence, remain open.

An attempt to tackle such questions in the case of plane Couette flow led us to derive a model in terms of three partial differential equations. ${ }^{15}$ Such a model has brought some elements of understanding to these problems, such as the nature of the flow on the outskirts of a turbulent $\operatorname{spot}^{16}$ and the spreading mechanism. ${ }^{17}$

This paper is devoted to the derivation and the study of such a model for the plane Poiseuille flow.

The outline of the paper is as follows. The model is first derived in Sec. II. Then some numerical results on the dynamics of turbulent spots are presented in Sec. III. The flow outside and inside the turbulent domain is analyzed, and waves at the wing tips are observed. The main results of this paper are assessed in Sec. IV.

\section{THE MODEL}

The Navier-Stokes equation and continuity condition for an incompressible flow read

$$
\partial_{t} \mathbf{v}+\mathbf{v} \boldsymbol{\nabla} \cdot \mathbf{v}=-\boldsymbol{\nabla} p+\nu \boldsymbol{\nabla}^{2} \mathbf{v},
$$




$$
\boldsymbol{\nabla} \cdot \mathbf{v}=0
$$

with $\mathbf{v} \equiv(u, v, w)$, where $u$ is the streamwise $(x), v$ is the wall-normal $(y)$, and $w$ is the spanwise $(z)$ velocity component, $p$ is the pressure, and $\nu$ is the kinematic viscosity. $\nabla^{2}$ denotes the three-dimensional Laplacian. In the following, we use dimensionless quantities. Lengths are scaled with the half-channel height $h$, and the centerline velocity $U_{\mathrm{cl}}$ stands for the velocity scale. Hence the velocity profile of the base flow, generated by a constant pressure gradient, is $U(y)=U_{b}\left(1-y^{2}\right)$ for $y \in[-1,1]$, where $U_{b}=1$ and the Reynolds number is $R=U_{\mathrm{cl}} h / \nu$. Equations (1) and (2) are further developed for the perturbations $\left(u^{\prime}, v^{\prime}, w^{\prime}, p^{\prime}\right)$ to the laminar flow and read

$$
\begin{aligned}
\partial_{t} u^{\prime} & +\left(\partial_{y} u^{\prime}-\partial_{x} v^{\prime}\right) v^{\prime}+\left(\partial_{z} u^{\prime}-\partial_{x} w^{\prime}\right) w^{\prime} \\
= & -\partial_{x} p^{\prime}-U \partial_{x} u^{\prime}-v^{\prime} \frac{d}{d y} U+R^{-1} \nabla^{2} u^{\prime}, \\
\partial_{t} v^{\prime} & +\left(\partial_{x} v^{\prime}-\partial_{y} u^{\prime}\right) u^{\prime}+\left(\partial_{z} v^{\prime}-\partial_{y} w^{\prime}\right) w^{\prime} \\
= & -\partial_{y} p^{\prime}-U \partial_{x} v^{\prime}+R^{-1} \nabla^{2} v^{\prime}, \\
\partial_{t} w^{\prime} & +\left(\partial_{x} w^{\prime}-\partial_{z} u^{\prime}\right) u^{\prime}+\left(\partial_{y} w^{\prime}-\partial_{z} v^{\prime}\right) v^{\prime} \\
= & -\partial_{z} p^{\prime}-U \partial_{x} w^{\prime}+R^{-1} \nabla^{2} w^{\prime}, \\
0= & \partial_{x} u^{\prime}+\partial_{y} v^{\prime}+\partial_{z} w^{\prime},
\end{aligned}
$$

where the nonlinear terms have been rewritten using the rotational form (see Appendix A).

\section{A. Expansions of the velocity components}

The Galerkin method is a special case of a weighted residual method. It consists of the separation of the in-plane $(x, z)$ and the wall-normal $(y)$ coordinates by expanding the perturbations $\left(u^{\prime}, v^{\prime}, w^{\prime}, p^{\prime}\right)$ onto a complete orthogonal basis of $y$-dependent functions satisfying the boundary conditions with amplitudes dependent on $(x, z, t)$. The equations of motion are then projected onto the same functional basis. The main modeling step is then performed when truncating these expansions at a low order to get a consistent and closed system governing the retained amplitudes. The projections are performed by taking the canonical scalar product $\langle.,$. defined by

$$
\langle f, g\rangle=\int_{-1}^{+1} f(y) g(y) d y .
$$

The no-slip boundary conditions for the wall-normal velocity component are

$$
\left.v^{\prime}\right|_{y= \pm 1}=\left.\partial_{y} v^{\prime}\right|_{y= \pm 1}=0
$$

obtained by combining the continuity equation (6) to the conditions

$$
\left.u^{\prime}\right|_{y= \pm 1}=\left.w^{\prime}\right|_{y= \pm 1}=0 .
$$

Hence, the wall-normal velocity is expanded as

$$
v^{\prime}(x, z, y, t)=\sum_{n \geq 1} V_{n}(x, z, t) S_{n}(y)
$$

where for an integer $n, S_{n}(y)=\left(1-y^{2}\right)^{2} Q_{n}(y)$, where $Q_{n}$ is an arbitrary polynomial. The first two polynomials are

$$
S_{1}(y)=C y\left(1-y^{2}\right)^{2} \quad \text { and } \quad S_{2}(y)=B\left(1-y^{2}\right)^{2},
$$

where $B$ and $C$ are constants. In the same way, the polynomials $R_{n}$ for the in-plane velocity $\left(u^{\prime}, w^{\prime}\right)$ have the form $R_{n}(y)=\left(1-y^{2}\right) T_{n}(y)$, where $T_{n}$ is an arbitrary polynomial. The first polynomial is $R_{0}(y)=\left(1-y^{2}\right)$. The second and the third ones are determined by the continuity equation through $R_{n}(y) \propto d / d y S_{n}(y), n=1,2$, and read

$$
R_{1}(y)=B\left(1-y^{2}\right)\left(5 y^{2}-1\right) \quad \text { and } \quad R_{2}(y)=F y\left(1-y^{2}\right) .
$$

The next step in the modeling is to introduce the truncated expansions into Eqs. (3)-(6) and to project over each polynomial using the scalar product (7).

The parity properties of the polynomials guarantee the orthogonality of the different contributions. Hence, the contribution of $R_{2}$ is separated from the contributions of $R_{0}$ and $R_{1}$. However, $R_{0}$ and $R_{1}$ have the same parity and hence their contributions are not separated. To remedy to this, we use the Gram-Schmidt orthogonalization method to construct $R_{0}$, which is orthogonal to $R_{1}$. With the polynomial $R_{0}(y)=A\left(1-y^{2}\right)\left(1+3 y^{2}\right)$, we have $\left\langle R_{0}, R_{1}\right\rangle=0$ and the considered expansions read

$$
u^{\prime}=U_{0}(x, z, t) R_{0}(y)+U_{1}(x, z, t) R_{1}(y)+U_{2}(x, z, t) R_{2}(y),
$$

$$
\begin{aligned}
& v^{\prime}=V_{1}(x, z, t) S_{1}(y)+V_{2}(x, z, t) S_{2}(y), \\
& w^{\prime}=W_{0}(x, z, t) R_{0}(y)+W_{1}(x, z, t) R_{1}(y)+W_{2}(x, z, t) R_{2}(y),
\end{aligned}
$$

$$
p^{\prime}=P_{0}(x, z, t) R_{0}(y)+P_{1}(x, z, t) R_{1}(y)+P_{2}(x, z, t) R_{2}(y),
$$

with the polynomials

$$
\begin{aligned}
& R_{0}(y)=A\left(1-y^{2}\right)\left(1+3 y^{2}\right), \\
& R_{1}(y)=B\left(1-y^{2}\right)\left(5 y^{2}-1\right), \\
& R_{2}(y)=F y\left(1-y^{2}\right), \\
& S_{1}(y)=C y\left(1-y^{2}\right)^{2}, \\
& S_{2}(y)=B\left(1-y^{2}\right)^{2},
\end{aligned}
$$

plotted in Fig. 1. The normalization constants are $A^{2}$ $=105 / 256, B^{2}=315 / 256, C^{2}=3465 / 256$, and $F^{2}=105 / 16$. The pressure components $P_{0}, P_{1}$, and $P_{2}$ introduce themselves as the Galerkin projection of the pressure $p^{\prime}$ on $R_{0}, R_{1}$, and $R_{2}$, respectively.

Next, by inserting the expansions (10)-(12) in the continuity equation (6) and projecting, respectively, on the polynomials $R_{0}, R_{1}$, and $R_{2}$, we get the three equations 

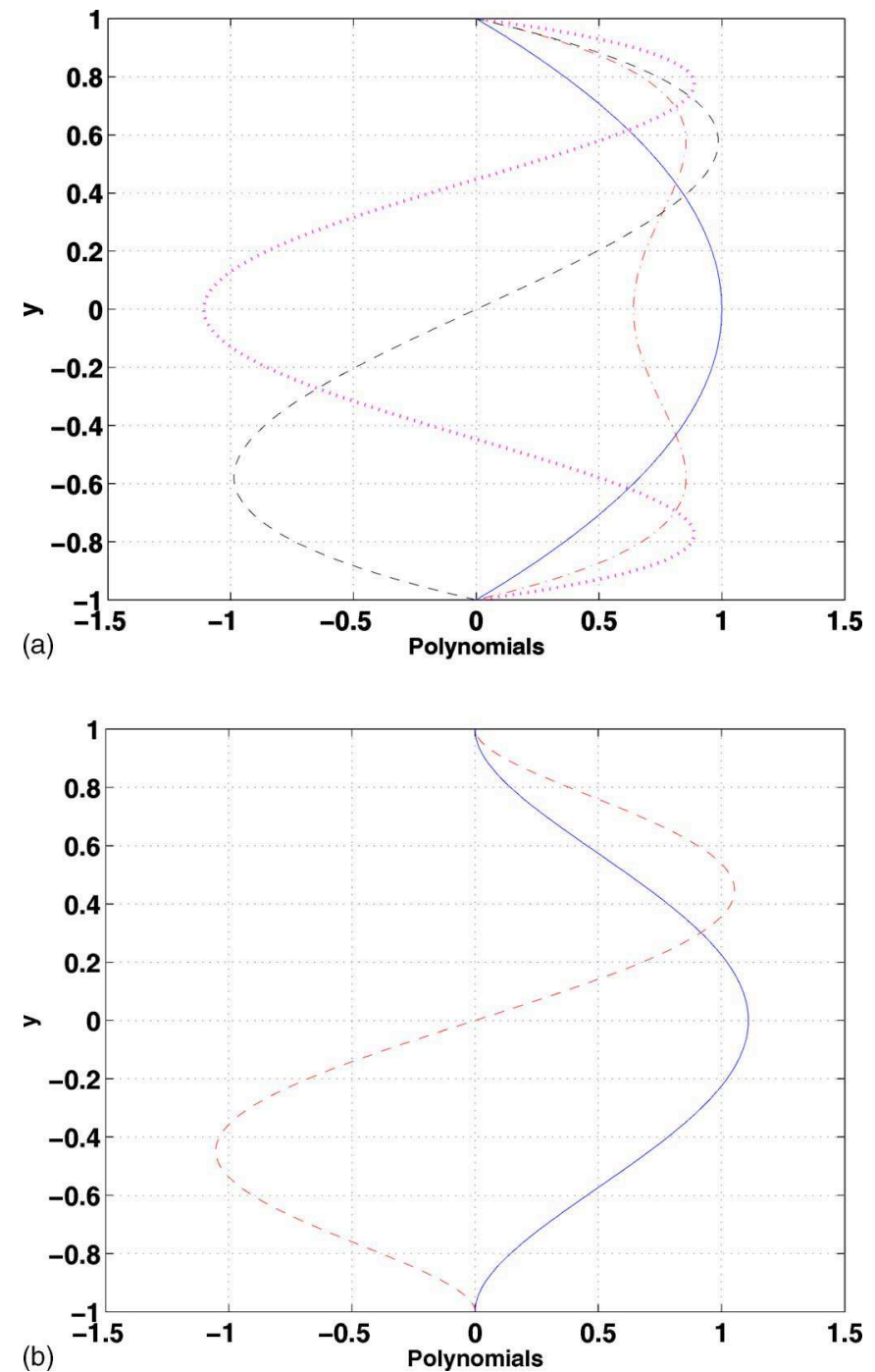

FIG. 1. (Color online) Profiles of the basis functions used in the derivation of the model. (a) Polynomials for the in-plane velocity. $R_{0}$ with dash-dotted (red) line, $R_{1}$ in dotted (magenta), $R_{2}$ in dashed (black), and the base flow $U(y)=U_{\mathrm{b}}\left(1-y^{2}\right)$ in solid (blue). (b) Polynomials for the wall-normal velocity. $S_{1}$ in dashed (red) and $S_{2}$ in solid (blue).

$$
\begin{aligned}
& \partial_{x} U_{0}+\partial_{z} W_{0}=0, \quad \partial_{x} U_{1}+\partial_{z} W_{1}=\beta_{1} V_{1}, \\
& \partial_{x} U_{2}+\partial_{z} W_{2}=\beta_{2} V_{2},
\end{aligned}
$$

where $\beta_{1}=\sqrt{11}$ and $\beta_{2}=\sqrt{3}$. Then from the projection of the Navier-Stokes equations (3)-(5) on the expansions (10)-(13), we get the equations of the amplitudes.

For $U_{0}$ and $W_{0}$, we have

$$
\begin{aligned}
\partial_{t} U_{0}+N_{U_{0}}= & -\partial_{x} P_{0}-b_{1} U_{b} \partial_{x} U_{0}+b_{2} U_{b} \partial_{x} U_{1}+\beta_{1} b_{3} U_{b} V_{1} \\
& -b_{4} U_{1} / R+R^{-1}\left(\Delta_{2}-\gamma_{0}\right) U_{0}, \\
\partial_{t} W_{0}+N_{W_{0}}= & -\partial_{z} P_{0}-b_{1} U_{b} \partial_{x} W_{0}+b_{2} U_{b} \partial_{x} W_{1}-b_{4} W_{1} / R \\
& +R^{-1}\left(\Delta_{2}-\gamma_{0}\right) W_{0},
\end{aligned}
$$

with $\gamma_{0}=\frac{11}{2}, \Delta_{2}=\partial_{x x}+\partial_{z z}$, and the nonlinear terms, noted $N_{U_{0}}$ and $N_{W_{0}}$, are given in Appendix B. In the same way, we obtain the equations of $U_{1}, W_{1}$, and $V_{1}$ :

$$
\begin{aligned}
\partial_{t} U_{1}+N_{U_{1}}= & -\partial_{x} P_{1}+b_{2} U_{b} \partial_{x} U_{0}-b_{1} U_{b} \partial_{x} U_{1}+U_{b 1} V_{1} / \beta_{1} \\
& -b_{4} U_{0} / R+R^{-1}\left(\Delta_{2}-\gamma_{1}\right) U_{1}, \\
\partial_{t} W_{1}+N_{W_{1}}= & -\partial_{z} P_{1}+b_{2} U_{b} \partial_{x} W_{0}-b_{1} U_{b} \partial_{x} W_{1}-b_{4} W_{0} / R \\
& +R^{-1}\left(\Delta_{2}-\gamma_{1}\right) W_{1}, \\
\partial_{t} V_{1}+N_{V_{1}}= & -\beta_{1} P_{1}-b_{5} U_{b} \partial_{x} V_{1}+R^{-1}\left(\Delta_{2}-\beta_{1}^{2}\right) V_{1}
\end{aligned}
$$

with $\gamma_{1}=\frac{45}{2}$, and the equations of $U_{2}, W_{2}$, and $V_{2}$ :

$$
\begin{aligned}
\partial_{t} U_{2}+N_{U_{2}}= & -\partial_{x} P_{2}+\frac{U_{b}}{\beta_{2}} V_{2}-\frac{2}{3} U_{b} \partial_{x} U_{2} \\
& +R^{-1}\left(\Delta_{2}-\gamma_{2}\right) U_{2}, \\
\partial_{t} W_{2}+N_{W_{2}}= & -\partial_{z} P_{2}-\frac{2}{3} U_{b} \partial_{x} W_{2}+R^{-1}\left(\Delta_{2}-\gamma_{2}\right) W_{2}, \\
\partial_{t} V_{2}+N_{V_{2}}= & -\beta_{2} P_{2}-\frac{10}{11} U_{b} \partial_{x} V_{2}+R^{-1}\left(\Delta_{2}-\beta_{2}^{2}\right) V_{2},
\end{aligned}
$$

with $\gamma_{2}=\frac{21}{2}$. The nonlinear terms $N_{U_{1}}, N_{W_{1}}, N_{V_{1}}, N_{U_{2}}, N_{W_{2}}$, and $N_{V_{2}}$ are given in Appendix B. Then, to eliminate the pressures $P_{0}, P_{1}$, and $P_{2}$ in Eqs. (15) and (16), Eqs. (17)-(19) and Eqs. (20)-(22), respectively, we introduce stream functions $\left(\Psi_{0}(x, z, t), \Psi_{1}(x, z, t), \Psi_{2}(x, z, t)\right)$ and velocity potentials $\left(\Phi_{1}(x, z, t), \Phi_{2}(x, z, t)\right)$ that satisfy the continuity equations,

$$
\begin{aligned}
& U_{0}=-\partial_{z} \Psi_{0}, \quad W_{0}=\partial_{x} \Psi_{0}, \\
& U_{1}=\partial_{x} \Phi_{1}-\partial_{z} \Psi_{1}, \quad W_{1}=\partial_{z} \Phi_{1}+\partial_{x} \Psi_{1}, \quad \beta_{1} V_{1}=\Delta_{2} \Phi_{1}, \\
& U_{2}=\partial_{x} \Phi_{2}-\partial_{z} \Psi_{2}, \quad W_{2}=\partial_{z} \Phi_{2}+\partial_{x} \Psi_{2}, \quad \beta_{2} V_{2}=\Delta_{2} \Phi_{2} .
\end{aligned}
$$

The equation governing $\Psi_{0}\left(\Psi_{1}\right.$ and $\left.\Psi_{2}\right)$ is obtained by cross-differentiating and subtracting the equations for the velocity components (15) and (16) [(17) and (18) and (20) and (21)] (the rotational part). Then, to derive the equation for the velocity potential $\Phi_{1}\left(\Phi_{2}\right)$, we take the divergence of Eqs. (17) and (18) [(20) and (21)], which yields an equation for the pressure $P_{1}\left(P_{2}\right)$, which is next used with Eq. (19) [Eq. (22)] to determine the potential part of the velocity field accounted for by the field $\Phi_{1}\left(\Phi_{2}\right)$. Finally, the five equations of the model are

$$
\begin{aligned}
{\left[\partial_{t}-R^{-1}\left(\Delta_{2}-\gamma_{0}\right)\right] \Delta_{2} \Psi_{0}=} & -b_{1} \partial_{x} \Delta_{2} \Psi_{0}+b_{2} \partial_{x} \Delta_{2} \Psi_{1} \\
& -b_{3} \partial_{z} \Delta_{2} \Phi_{1}-b_{4} \Delta_{2} \Psi_{1} / R \\
& +\left(\partial_{z} N_{U_{0}}-\partial_{x} N_{W_{0}}\right), \\
{\left[\partial_{t}-R^{-1}\left(\Delta_{2}-\gamma_{1}\right)\right] \Delta_{2} \Psi_{1}=} & -b_{1} \partial_{x} \Delta_{2} \Psi_{1}+b_{2} \partial_{x} \Delta_{2} \Psi_{0} \\
& -b_{4} \Delta_{2} \Psi_{0} / R-\partial_{z} \Delta_{2} \Phi_{1} / \beta_{1}^{2} \\
& +\left(\partial_{z} N_{U_{1}}-\partial_{x} N_{W_{1}}\right),
\end{aligned}
$$



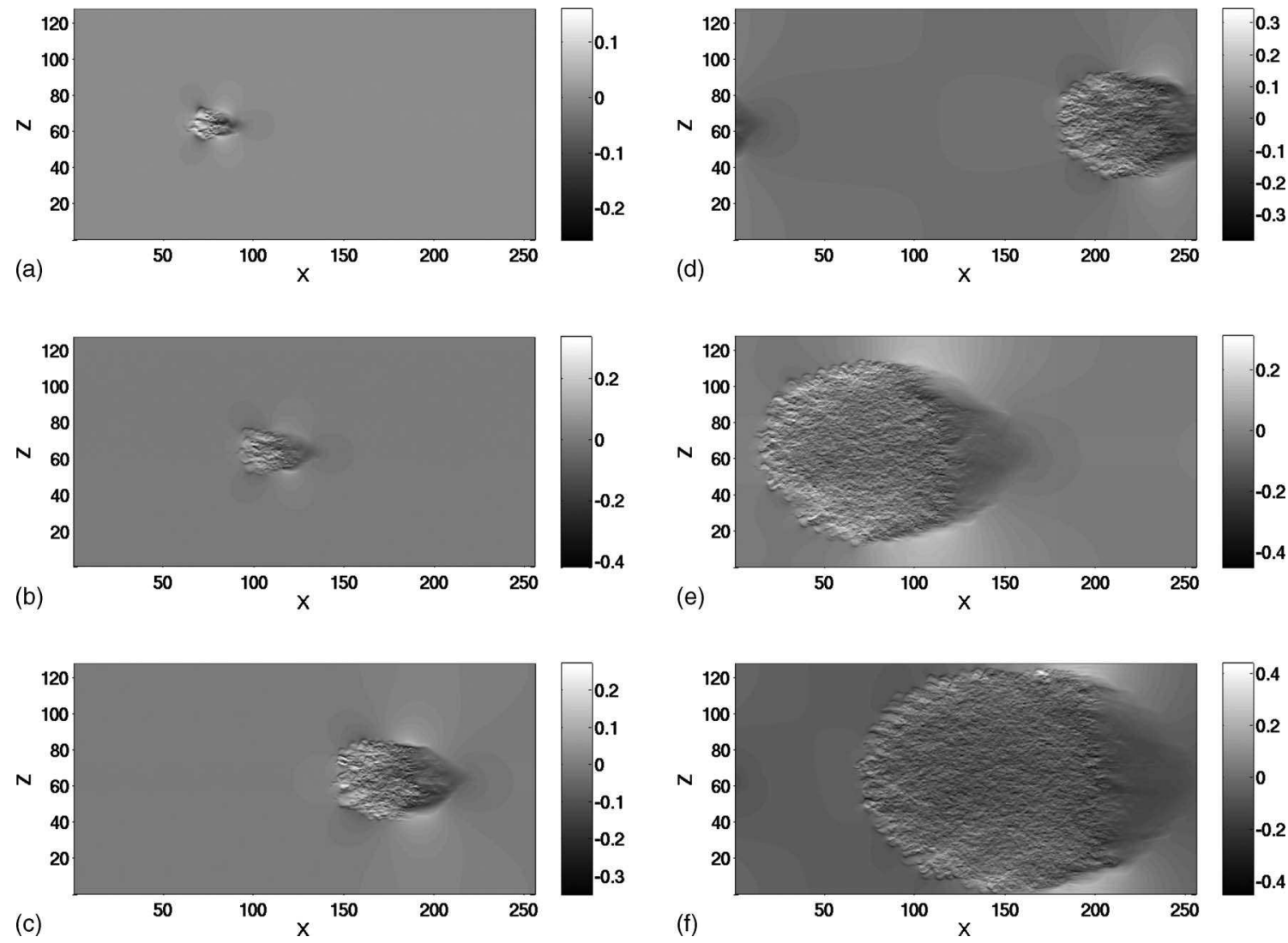

FIG. 2. Evolution of a turbulent spot depicted by the streamwise velocity $U_{0} . R=900$. From top to bottom and left to right: $t=64,112,208,272,448$, and 560 (enhanced online).

$$
\begin{aligned}
{\left[\partial_{t}-\right.} & \left.R^{-1}\left(\Delta_{2}-\beta_{1}^{2}\right)\right]\left(\Delta_{2}-\beta_{1}^{2}\right) \Delta_{2} \Phi_{1} \\
= & \beta_{1}^{2}\left(\partial_{x} N_{U_{1}}+\partial_{z} N_{W_{1}}\right)-\beta_{1} \Delta_{2} N_{V_{1}}+\frac{23 \beta_{1}^{2}}{2 R} \Delta_{2} \Phi_{1} \\
& +\left(7-b_{5} \Delta_{2}\right) \partial_{x} \Delta_{2} \Phi_{1}, \\
& \quad+\left(\partial_{z} N_{U_{2}}-\partial_{x} N_{W_{2}}\right), \\
{\left[\partial_{t}-R^{-1}\left(\Delta_{2}-\gamma_{2}\right)\right] \Delta_{2} \Psi_{2}=} & -\frac{2}{3} \partial_{x} \Delta_{2} \Psi_{2}-\partial_{z} \Delta_{2} \Phi_{2} / \beta_{2}^{2} \\
= & \left.\left.\beta_{2}^{2}\left(\partial_{2}-\beta_{2}^{2}\right)\right]\left(\Delta_{2}-\beta_{2}^{2}\right) \Delta_{2} \Phi_{2} \partial_{z} N_{W_{2}}\right)-\beta_{2} \Delta_{2} N_{V_{2}}+\frac{15 \beta_{2}^{2}}{2 R} \Delta_{2} \Phi_{2} \\
& +\left(1-\frac{10}{11} \Delta_{2}\right) \partial_{x} \Delta_{2} \Phi_{2} .
\end{aligned}
$$

\section{NUMERICAL SIMULATIONS}

A standard Fourier pseudospectral method with periodic boundary conditions in the streamwise $(x)$ and spanwise $(z)$ directions has been used for the integration of the equations of the model (26)-(30). A second-order Adams-Bashforth scheme is used for the advancement in time. Throughout the paper, numerical simulations are performed in a computational box with streamwise and spanwise lengths $L_{x} \times L_{z}$ $=256 \times 128$. The spatial resolution is $d x=d z=0.125$ and the time step is $d t=0.01$. For the considered value of the Reynolds number $(R=900)$, the obtained results are quite the same for higher resolutions $(d x=d z=0.1, d x=d z=0.05)$ and smaller time step $(d t=0.001)$.

For the initial condition, we take the localized functions,

$$
\begin{aligned}
& \Psi_{n}(x, z, t=0)=A \exp ^{-\left(x^{2}+z^{2}\right) / \sigma}, \quad n=0,1,2, \\
& \Phi_{n}(x, z, t=0)=0, \quad n=1,2,
\end{aligned}
$$

where $A=1$ is an amplitude and $\sigma=2$ is related to the size of the initial turbulent domain.

For $R \leq 850$, all the initial turbulent states decayed, whereas for $R \geq 900$, growing spots have been obtained. Hence, the stability threshold $R_{\mathrm{g}}$ for this model is between 850 and 900 . We did not determine the value of $R_{\mathrm{g}}$ with more precision, since the aim of this paper is to study the features of the turbulent spot and to focus on the mechanisms at work.

The spatiotemporal evolution of a turbulent spot can be illustrated by one of its velocity components. ${ }^{19}$ From a qualitative point of view, the evolution depicted by the total streamwise velocity (base flow + perturbations) at the channel centerline is similar to that depicted by the component $U_{0}$, plotted at different times in Fig. 2. The interior of the spots shows $x$-elongated patches of $U_{0}$ with $z$-alternating sign. The physical interpretation of these patches is postponed for future work. One can already note that a positive 

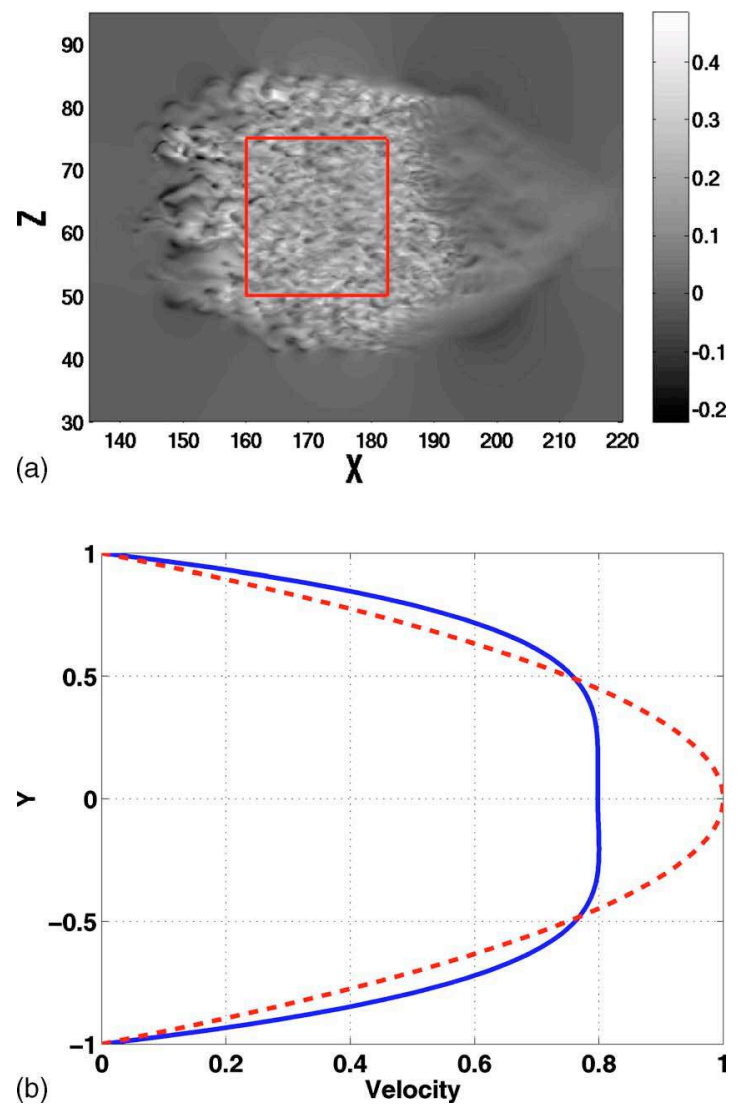

FIG. 3. (Color online) (a) Spatial distribution of $U_{1}$. In the turbulent region limited by the (red) box, $U_{1}$ is mainly positive. (b) The mean profile of the total streamwise velocity (in solid blue). The laminar parabolic profile is shown with the dashed (red) line.

$U_{0}$ represents a high-speed streak since $U(y)+U_{0} R_{0}(y)$ $\geq U(y)$ and a negative $U_{0}$ represents a low-speed streak since $U(y)+U_{0} R_{0}(y) \leq U(y)$.

The general shape and the evolution of the spot compare well with the visualizations of both Carlson et al. ${ }^{1}$ and Alavyoon et $a .^{2}$ At an early stage, the spot has an arrowhead front part $(t=112)$. Then, as time proceeds, a disturbed laminar-like region develops at its leading edge (e.g., $x$ $\geq 200$ at $t=560$ ) and the spot gets a turbulent crescent shape.

In the following, some characteristics of the turbulent spot are presented, showing a qualitative consistency between previous experimental results and our own numerical simulations of the model.

\section{A. Mean profiles}

Figure 3 shows the spot at $t=208$ depicted by the velocity component $U_{1}$. The turbulent region is clearly separated from the disturbed laminar-like region in front of the spot (for $x \geq 190$ ). This turbulent region is limited by the red-box and we define the mean value of any velocity component $Z$ over the surface $\mathcal{S}$ of this box by

$$
\bar{Z}=\int_{\mathcal{S}} Z d x d z / \mathcal{S} .
$$

The mean profile of the total streamwise velocity $U(y)+\overline{u^{\prime}}=U(y)+\overline{U_{0}} R_{0}+\overline{U_{1}} R_{1}+\overline{U_{2}} R_{2}$ is given in Fig. 3(b). It
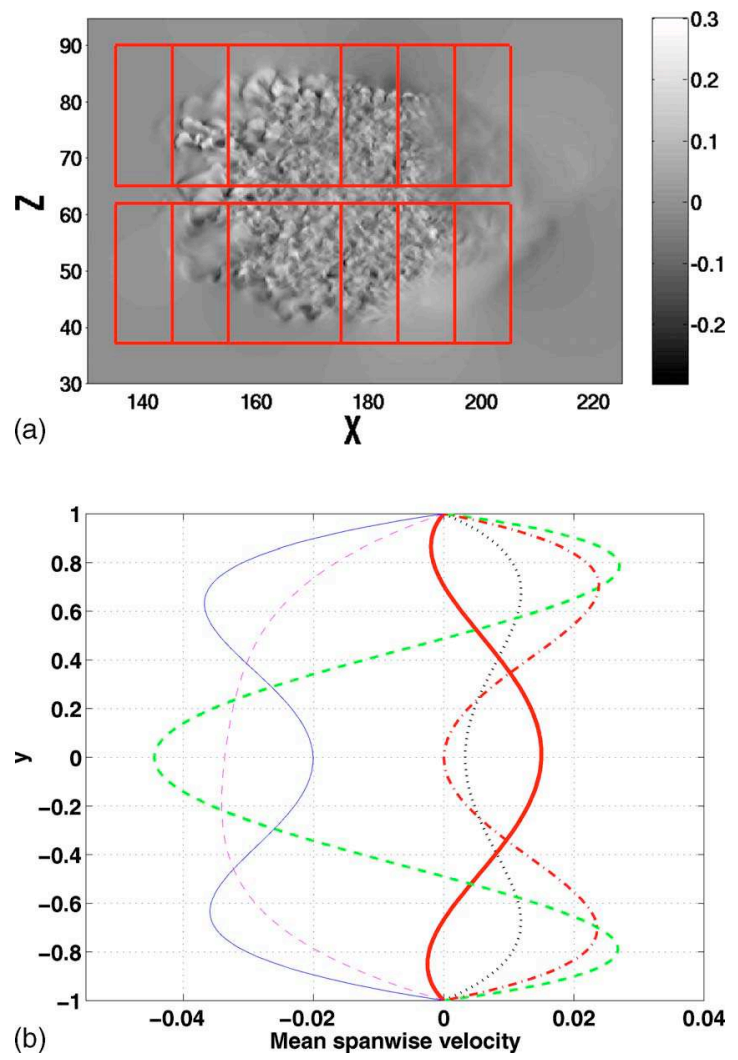

FIG. 4. (Color online) (a) The distribution of the spanwise velocity $W_{1}$. The spot is split into small (arbitrary) regions limited by (red) boxes. There are six boxes for $z \geq 64$ and six others for $z \leq 64$. (b) The mean value of the total spanwise velocity in each bottom box $(z \leq 64)$ : From left to right: box number (1) with a dotted (black) line, (2) with a thick solid (red) line, (3) with a thin solid (blue) line, (4) with a thin dashed (magenta) line, (5) with a thick dashed (green) line, and (6) with a thin dash-dotted (red) line.

is similar to the turbulent profile of $\mathrm{pPf}$ and this is mainly due to the positiveness of $\overline{U_{1}}$ within the turbulent region, $\overline{U_{1}}=0.1650$, whereas the mean values of the other velocity components are one order smaller, $\overline{U_{0}}=-0.029$ and $\overline{U_{2}}=-0.0021$. Hence, the spot behaves as a localized region where the laminar flow is decelerated near the center $\left[U(y)+\overline{U_{1}} R_{1}(y) \leq U(y)\right]$ and accelerated near the walls $\left[U(y)+\overline{U_{1}} R_{1}(y) \geq U(y)\right]$.

Furthermore, Fig. 4 shows the same spot depicted by another velocity component, $W_{1}$. The mean values of the total spanwise velocity [Eq. (12)] over the boxes shown in Fig. 4(a) are given in Figs. 4(b) and 5(a). At different locations, the spanwise velocity has inflectional profiles similar to those used in Ref. 13 and shown, through a linear stability calculation, to be potentially unstable to oblique waves. Such waves are now studied.

\section{B. The waves at the wing tips of the spot}

One of the most interesting features of the spots in $\mathrm{pPf}$ is the presence of oblique waves at the wing tips of the spots. As shown in Fig. 5(b), such waves are easily observed by reporting the wall-normal velocity on the channel centerline. At this $y$ location, the wall-normal velocity given by Eq. (11) 

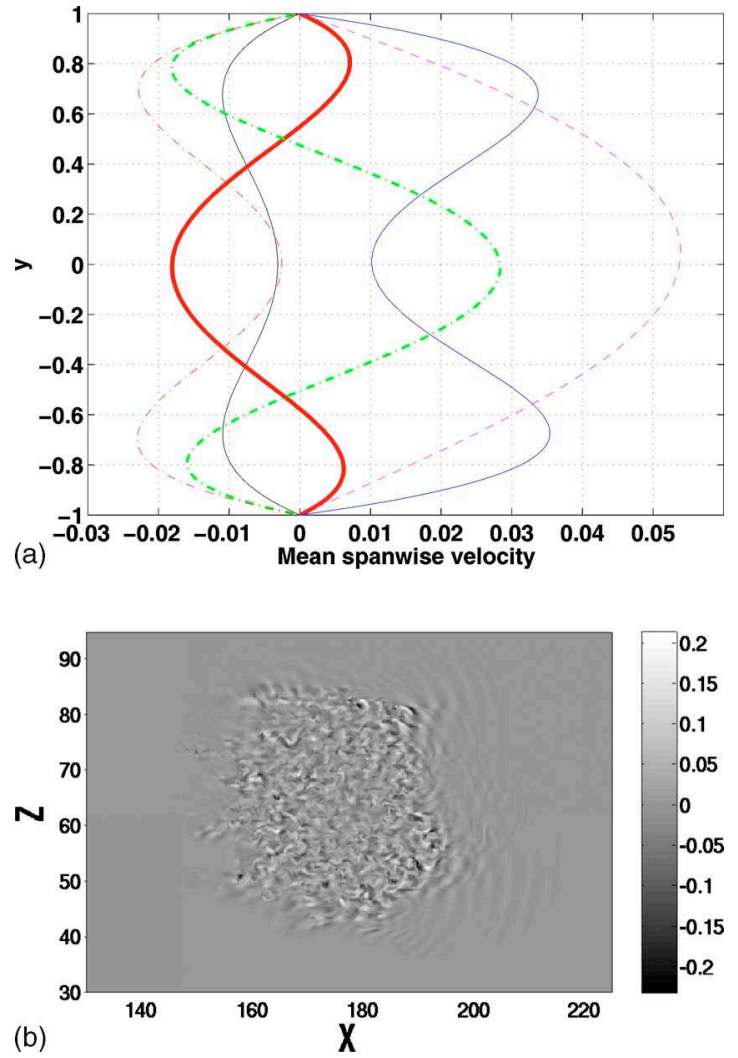

FIG. 5. (Color online) (a) The mean value of the total spanwise velocity in each upper box $(z \geq 64)$, shown in Fig. 4. Same color convention as in Fig. 4(b). (b) The spatial distribution of $V_{2}$. At the channel centerline, the total wall-normal velocity is equal to $B V_{2}$. Oblique waves are present at the wing tips of the spot (enhanced online).

reduces to $B V_{2}$. These waves can also be represented by the in-plane velocity component associated with $V_{2}$, i.e., $W_{2}$ and $U_{2}$, and are displayed in Fig. 6 .

The profiles of $U_{2}, W_{2}$, and $V_{2}$ as a function of the streamwise coordinate $(x)$ for $z=44$ and $z=86$ are given in Fig. 7. These plots clearly point out the wavy character of the velocity components $U_{2}, W_{2}$, and $V_{2}$ at the wing tip regions. On the one hand, the wavelength of these waves is about $5 h$ and is of the same order as the one measured by Alavyoon et al., ${ }^{2}$ where it is about two channel heights (i.e., 4h). On the other hand, regarding the magnitude of these waves, Henningson ${ }^{13}$ found an amplitude of $15 \%$ of $U_{\mathrm{cl}}$ for the wallnormal velocity component at the wing tips of the spot. In the present results, the amplitude is about $5 \%$ of $U_{\mathrm{cl}}$ $(B \sim 1.1)$.

Furthermore, Henningson and Alfredsson ${ }^{14}$ have investigated these wave packets by hot-film anemometry and have found that the streamwise velocity disturbance associated with these waves is antisymmetric with respect to the channel centerline (Fig. 7 in Ref. 14). This noteworthy point is in accordance with our results, since the $y$ dependence of the streamwise velocity $U_{2}$, associated with these waves, is odd in $y$ and is given by the polynomial $R_{2}(y)=F y\left(1-y^{2}\right)$.

As a conclusion, the waves packets located at the wing tips of the turbulent spot have a wall-normal velocity with a symmetric $y$ distribution and streamwise and spanwise velocities with an antisymmetric $y$ distribution. Thus, they have
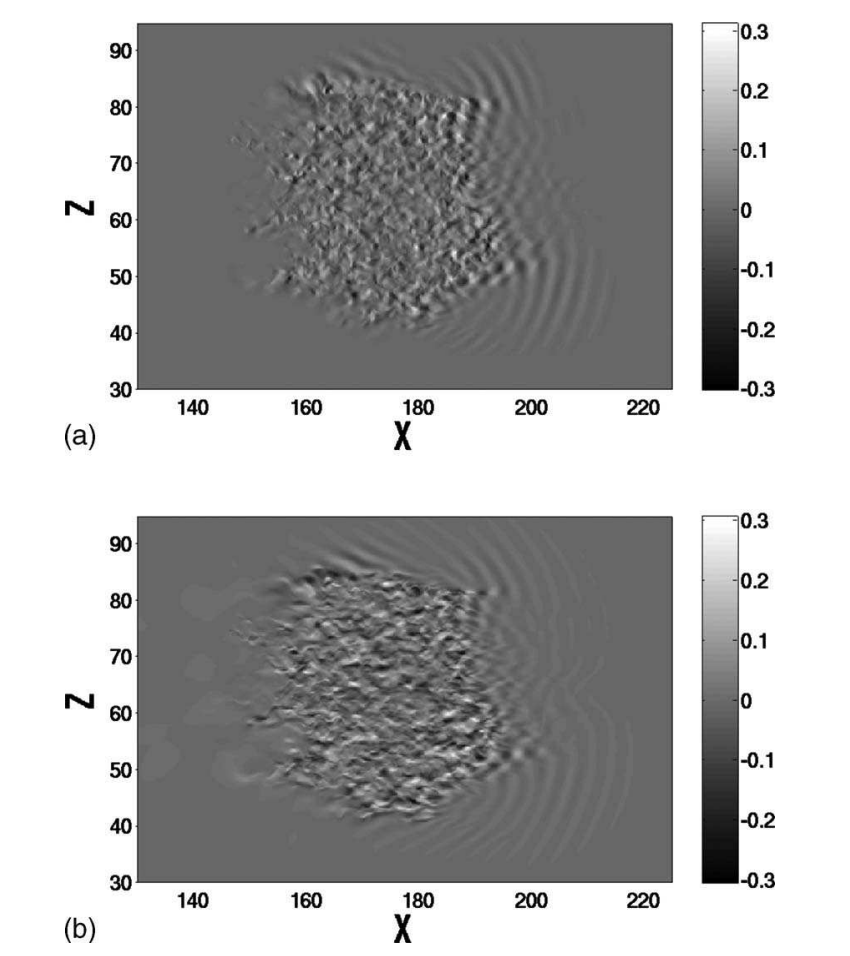

FIG. 6. The spatial distributions of the spanwise velocity $W_{2}$ (a) and of the streamwise velocity $U_{2}$ (b) associated with $V_{2}$ show waves at the wing tips of the spot. Not all the points are represented (enhanced online).

the same properties as the waves investigated by Henningson and Alfredsson ${ }^{14}$ and Henningson and Kim. ${ }^{18}$

\section{Large-scale flow around the spot}

Studying the spanwise velocity at the channel centerline, Henningson and $\mathrm{Kim}^{18}$ observed that there is a mean motion out from the spot toward the wing tips and that this outward motion continues in the laminar flow outside the wing tips, whereas there is a motion toward the spot downstream of the turbulent area. Near the walls, they also observed that the spanwise velocity exhibits the same feature as it does at the midplane. This particular distribution indicates that the flow around the spot is characterized by an outflow from the spot in the spanwise direction extending all over the gap and by a streamwise inflow toward the spot.

Within our modeling approach, this inflow is represented by the streamwise velocity component $U_{0} R_{0}(y)$ and this outflow by the spanwise component $W_{0} R_{0}(y)$. In fact, as shown in Fig. 8, on the leading edge of the spot $(x \geq 190), U_{0}$ is mainly negative, whereas on the trailing edge $(x \leq 150)$ it is mainly positive. Hence, this distribution points out the inflow character of the streamwise component $U_{0}$. To this inflow motion toward the spot corresponds an outflow motion represented by two regions where $W_{0}$ is mainly positive (for $z$ $\geq 70$ ) and negative (for $z \leq 50$ ), as shown in Fig. 8 .

By combining these features of the spanwise outflow and the streamwise inflow, we obtain a quadrupolar flow, shown in Fig. 9(a), similar to that observed around a turbulent spot in the plane Couette flow. ${ }^{7}$ The motion toward the spot downstream of the turbulent area [i.e., $x \geq 190$, in Fig. 9(a)] is more important than the motion toward the spot upstream. 

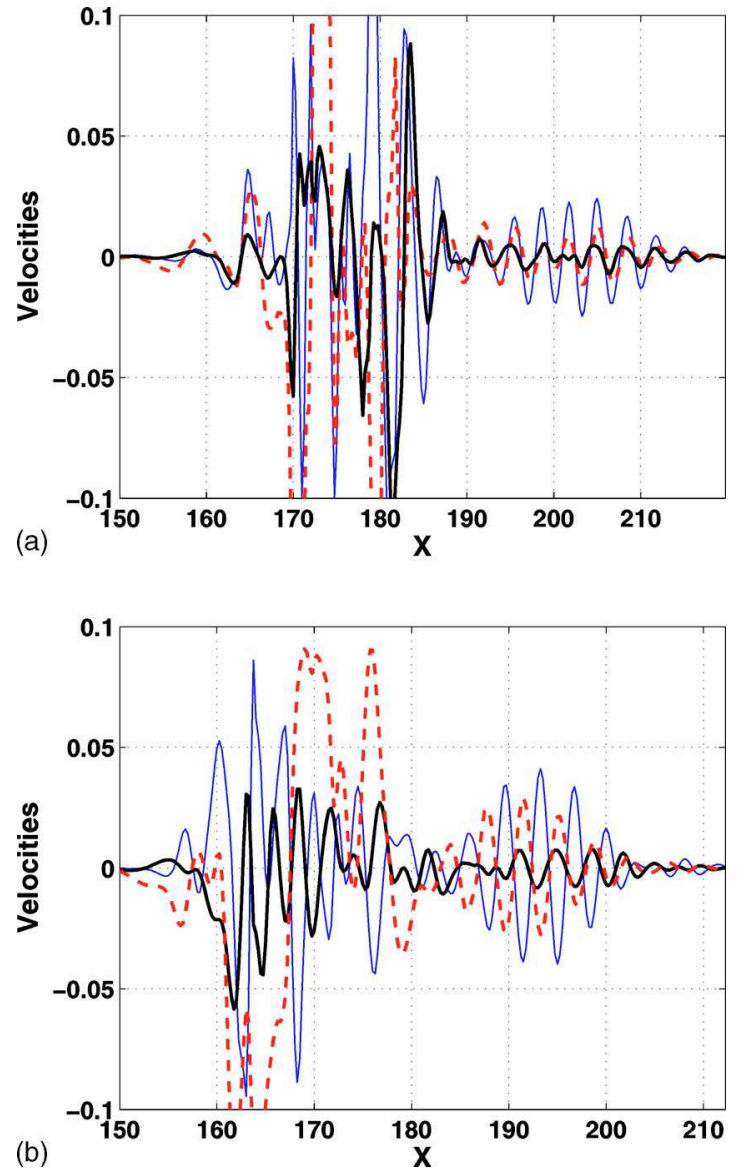

FIG. 7. (Color online) The profiles of $U_{2}$ (in dashed red), $W_{2}$ (in thin blue), $V_{2}$ (in thick black) along a streamwise line for $z=44$ (a) and $z=86$ (b). The wave packets are clearly observed for $x \in[190,215]$ (a) and $x \in[180,210]$ (b).

This quadrupolar structure can be already seen from the distribution of the stream function $\Psi_{0}$ associated with the flow $\left(U_{0}, W_{0}\right)$. This distribution, displayed in Fig. 9(b), is characterized by four lobes with alternating sign. Each lobe corresponds to a region of recirculation extending outside the spot.

The origin of this quadrupolar large-scale flow in the case of pCf was traced back to the positiveness of the Reynolds stress within the turbulent region. ${ }^{16}$ This may provide the starting point for a similar investigation in the present case.

\section{Coherent structures}

The interior structure of the turbulent domain is now investigated. A particular interest is given to the streamwise vortices, which play an important role in the energy production and hence in the sustainment of the turbulent state.

First, the Reynolds-Orr equation governs the time evolution of the perturbations energy $E(t)=\frac{1}{2} \int_{\mathcal{V}}\left(u^{\prime 2}+v^{\prime 2}\right.$ $\left.+w^{\prime 2}\right) d \mathcal{V}$, where $\mathcal{V}$ is the volume of the domain. If $P$ denotes the energy production issued from the interaction of the perturbation with the base flow $U(y), P=\int_{\mathcal{V}}-u^{\prime} v^{\prime} \frac{d}{d y} U d \mathcal{V}$, and if $D$ is the dissipation due to viscous effects, then this equation is written as $\frac{d}{d t} E=P-D$. The product $-u^{\prime} v^{\prime}$ is the Reynolds
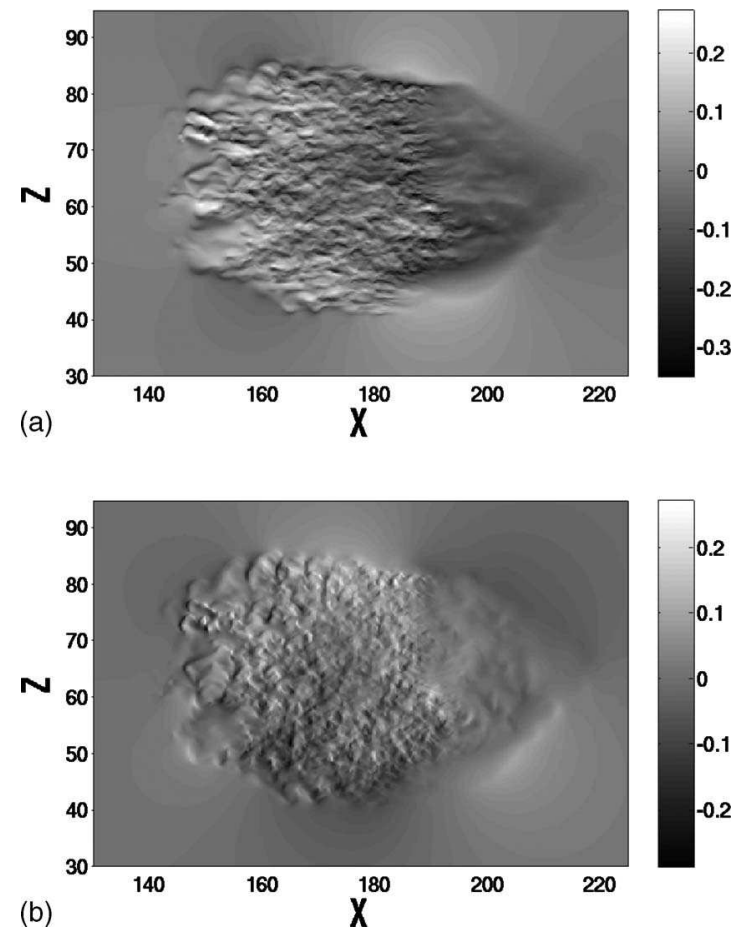

FIG. 8. The spatial distribution of $U_{0}$ (a) represents an inflow toward the spot, whereas $W_{0}$ (b) represents an outflow from the spot. Outside the spot, the in-plane flow $\left(U_{0}, W_{0}\right)$ is quadrupolar, as shown in Fig. 9.

stress component associated with the energy production. Within our modeling approach, by integrating over the gap, the production reads

$$
P=\int_{\mathcal{S}} \frac{5}{\sqrt{33}} U_{0} V_{1}+\frac{1}{\sqrt{11}} U_{1} V_{1}+\frac{1}{\sqrt{3}} U_{2} V_{2} d \mathcal{S},
$$

where $\mathcal{S}$ is the surface of the domain.

Second, the redistribution of the base flow by the wallnormal velocity associated with the streamwise vortices generates the streaks. This mechanism, called the lift-up effect, is represented by the linear term $-v^{\prime} \frac{d}{d y} U$ in Eq. (3). The linear term $\beta_{1} b_{3} U_{b} V_{1}$ in the $U_{0}$ equation comes from the Galerkin projection over $R_{0}$ of the lift-up term and accounts for the generation of the streak $U_{0}$ by the wall-normal velocity $V_{1}$. Regions where the lift-up effect occurs are hence characterized by positive Reynolds stress $U_{0} V_{1}$.

The inspection of the full turbulent domain, localized or filling the whole computational box, shows that the streamwise vortices are numerous. They are easily observed by monitoring the wall-normal velocity $V_{1}$, as shown in Fig. 10. The negative crescent contour of $V_{1}$ represents a flow going from the bottom wall $(y=-1)$ to the channel centerline $(y$ $=0)$ since $V_{1} S_{1}(y) \geq 0$. Then, it goes back toward the bottom wall (where $V_{1} \geq 0$ ) through the in-plane motion represented by the flow field $\left(U_{1}, W_{1}\right) R_{1}(y)$ [Fig. 10(b)]. This particular motion represents a crescent vortex in the half-space $y \in[-1,0]$, as shown in Fig. 11. Due to the asymmetry of the polynomial $S_{1}$ and to the symmetry of the polynomial $R_{1}$, a similar crescent vortex exists in the other half-space $y$ $\in[0,1]$, but rotating in the opposite sense. The two counterrotating streamwise vortices forming the legs of the crescent 


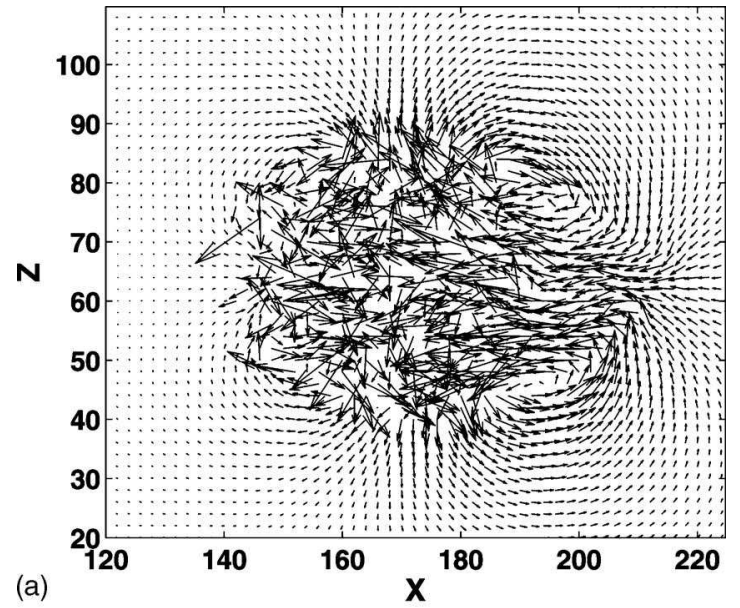

(a)

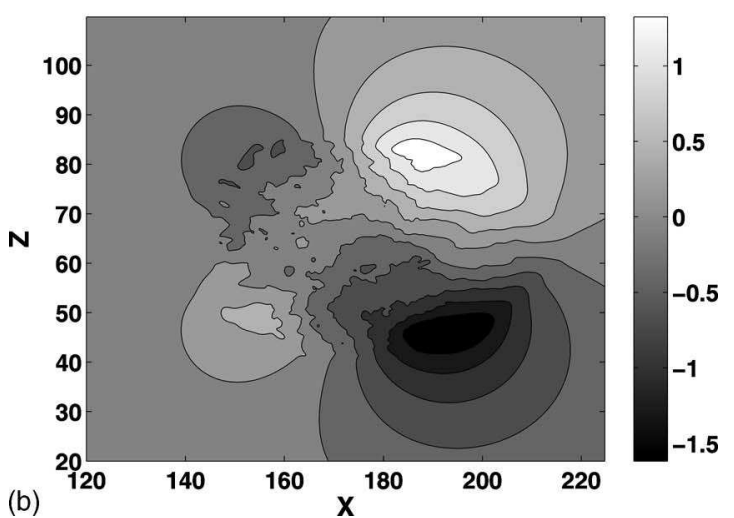

FIG. 9. (a) The flow field $\left(U_{0}, W_{0}\right)$ exhibits a quadrupolar structure (not all the points are represented). (b) Spatial distribution of the stream function $\Psi_{0}$. Outside the spot, it has four lobes with alternating sign. Each lobe corresponds to a region of large recirculation.

vortex regenerate the streaks by the lift-up effect: regions with positive (negative) $V_{1}$ in Fig. 10 correspond to regions of positive (negative) $U_{0}$ in Fig. 12. Thus, on each side of the legs, we have positive Reynolds stress $U_{0} V_{1}$ as shown in Fig. $12(\mathrm{~b})$.

Note that the flow field $\left(U_{0}, W_{0}\right)$, shown in Fig. 12, has some dipoles, which have been shown in Ref. 17 to be at the origin of the spreading of the turbulent domain through their self-advection.

Following the study of Papavassiliou and Hanratty, ${ }^{20}$ a comparison between the geometries of the streamwise vortices in the plane Couette and Poiseuille flows for lowReynolds numbers as well as their generation process will be presented in a forthcoming paper.

\section{CONCLUSION}

In this paper, we have derived a model in terms of five partial differential equations for pPf. The numerical simulation of the turbulent spot shows many features in close agreement with the results in the literature, supporting hence the modeling approach.

The spot consists of three distinct regions: a turbulent area, a disturbed area, and a wave area. Inside the turbulent area, the laminar flow is accelerated near the walls, whereas it is decelerated near the center of the channel, getting a flat
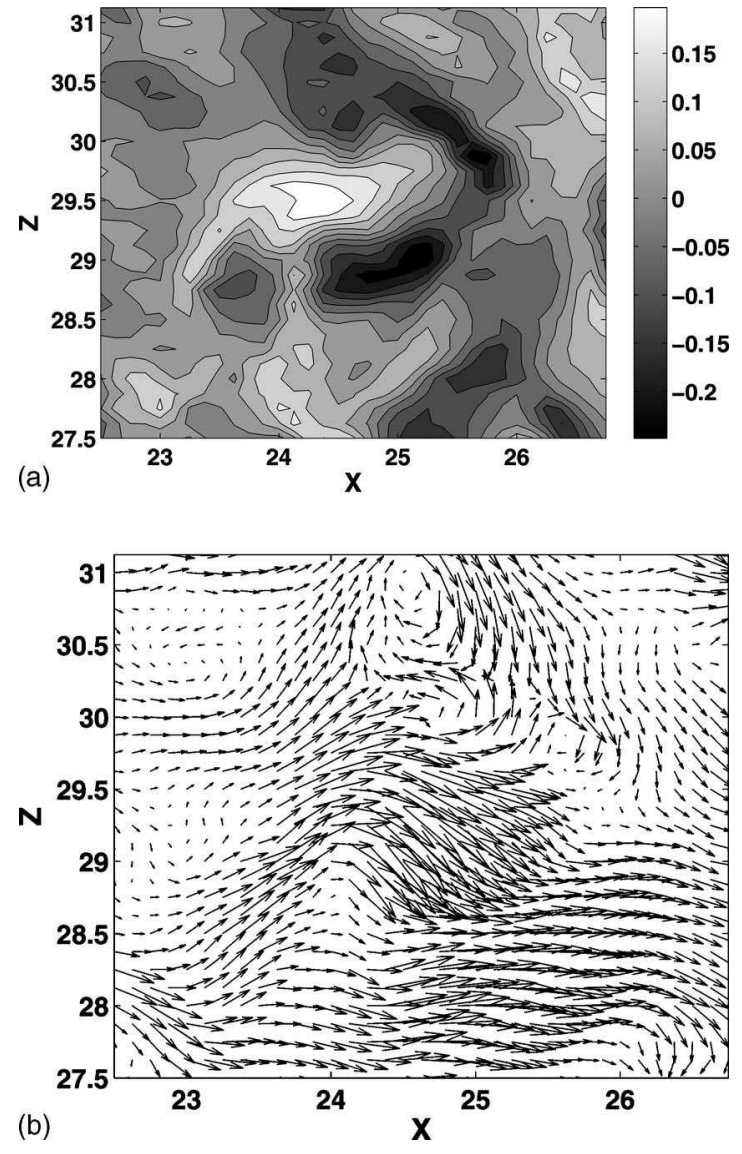

FIG. 10. Spatial distribution of $V_{1}$ (a) and the flow field $\left(U_{1}, W_{1}\right)$ (b). This spatial distribution represents a crescent vortex in each half-space $y \in[-1,0]$ and $y \in[0,1]$. A 3D representation is given in Fig. 11. The two legs of the crescent vortex are elongated in the streamwise direction and form the streamwise vortices.

shape typical of the turbulent plane Poiseuille flow. The oblique waves, observed at the wing tip regions, share several features with those studied in the literature, especially their velocity structure. They have a symmetric wall-normal velocity $\left(V_{2} S_{2}\right)$ and an antisymmetric streamwise velocity $\left(U_{2} R_{2}\right)$ with respect to the channel centerline. The origin of

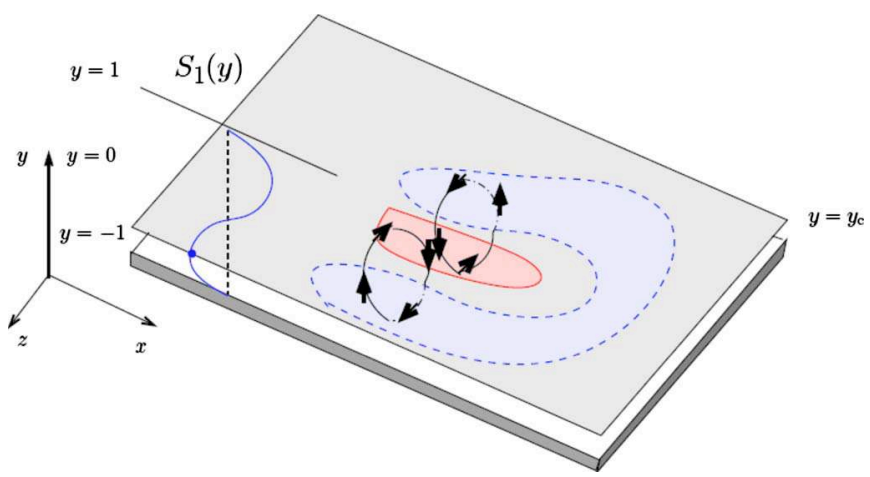

FIG. 11. (Color online) 3D reconstruction of the crescent vortex, depicted by $V_{1}$ and $\left(U_{1}, W_{1}\right)$ in Fig. 10. The distribution of $V_{1}$ has a crescent shape in the shaded plane for some $y=y_{\mathrm{c}} \in[0,-1]$. The two circles represent the cross section $(z, y)$ of the streamwise vortices. The polynomial $S_{1}$ is plotted by a solid blue line. For clarity, only the two counter-rotating streamwise vortices spanning the half-space $y \in[-1,0]$ are represented. 

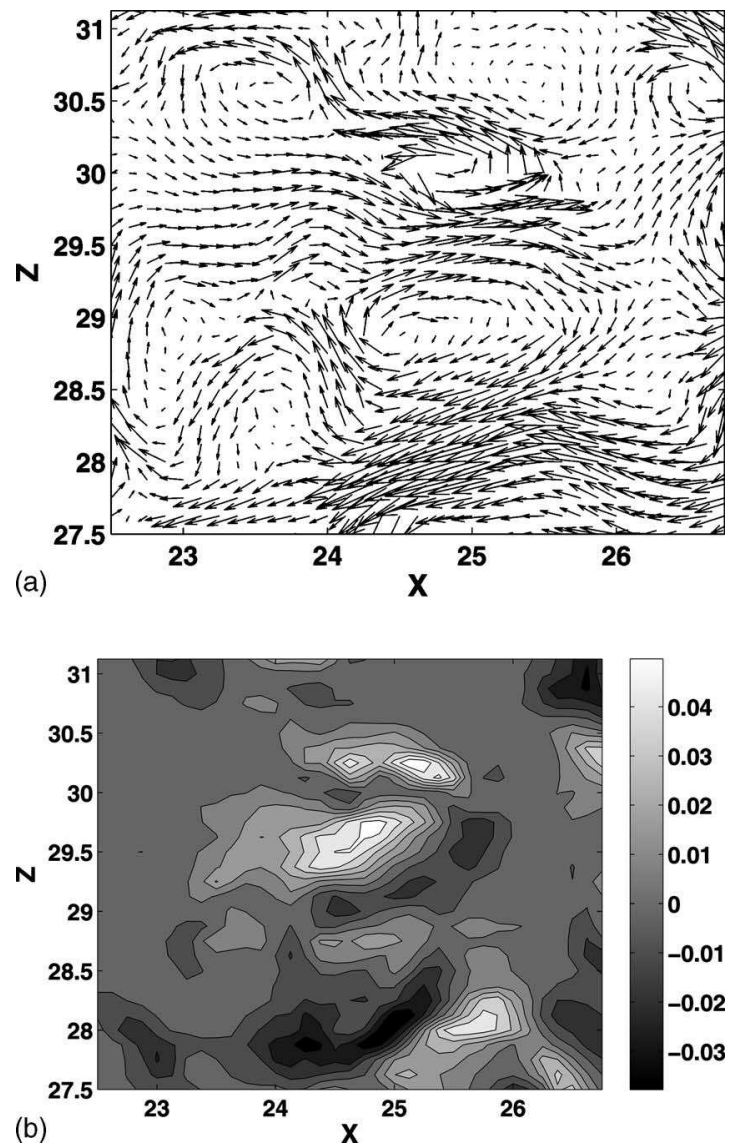

FIG. 12. (a) The flow field $\left(U_{0}, W_{0}\right)$ depicted by vectors. Two dipoles are clearly observed. (b) The Reynolds stress $U_{0} V_{1}$. There are three $x$-elongated patches with positive value.

these waves can be studied using this model, which will provide some elements of understanding on their nature and their role. One can already note that they contribute (albeit weakly) to the energy production because at the wing tips, the Reynolds stress $U_{2} V_{2}$ [in Eq. (31)] is positive, since $U_{2}$ and $V_{2}$ evolve in phase, as inferred from Fig. 7.

In conclusion, the model captures all essential features of the spot, such as its shape, its mean turbulent characteristics, and the presence of waves on the wing tips. This is an indication that further information that may be extracted from the model will be fruitful.

\section{APPENDIX A: THE EQUATIONS FOR THE PERTURBATIONS}

By expanding the velocity components $u=U(y)+u^{\prime}, v$ $=v^{\prime}, w=w^{\prime}$, and the pressure $p=\widetilde{p}+P^{0}$, where $P^{0}$ is the driving pressure $\left(\frac{d}{d x} P^{0}=\right.$ const $)$ and $\left(u^{\prime}, v^{\prime}, w^{\prime}, \widetilde{p}\right)$ are the perturbations, and inserting these expansions in Eqs. (1) and (2), we get

$$
\begin{aligned}
\partial_{t} u^{\prime} & +u^{\prime} \partial_{x} u^{\prime}+v^{\prime} \partial_{y} u^{\prime}+w^{\prime} \partial_{z} u^{\prime} \\
& =-\partial_{x} \tilde{p}-U \partial_{x} u^{\prime}-v^{\prime} \frac{d}{d y} U+R^{-1} \nabla^{2} u^{\prime},
\end{aligned}
$$

$$
\begin{aligned}
& \partial_{t} v^{\prime}+u^{\prime} \partial_{x} v^{\prime}+v^{\prime} \partial_{y} v^{\prime}+w^{\prime} \partial_{z} v^{\prime} \\
& =-\partial_{y} \tilde{p}-U \partial_{x} v^{\prime}+R^{-1} \nabla^{2} v^{\prime}, \\
& \partial_{t} w+u^{\prime} \partial_{x} w^{\prime}+v^{\prime} \partial_{y} w^{\prime}+w^{\prime} \partial_{z} w^{\prime} \\
& =-\partial_{z} \tilde{p}-U \partial_{x} w^{\prime}+R^{-1} \nabla^{2} w^{\prime}, \\
& 0=\partial_{x} u^{\prime}+\partial_{y} v^{\prime}+\partial_{z} w^{\prime} .
\end{aligned}
$$

Then, using the rotational form for the nonlinearities and defining $p^{\prime}=\widetilde{p}+E_{\mathrm{loc}}^{\prime}$ with $E_{\mathrm{loc}}^{\prime}=\frac{1}{2}\left(u^{\prime 2}+v^{\prime 2}+w^{\prime 2}\right)$, we get Eqs. (3)-(6).

\section{APPENDIX B: THE NONLINEAR TERMS OF THE MODEL}

$$
\begin{aligned}
& N_{U_{0}}=\alpha_{01} U_{0} V_{1}-\alpha_{06} V_{1} \partial_{x} V_{1}+\alpha_{02} U_{1} V_{1} \\
& +\alpha_{03} W_{0}\left(\partial_{z} U_{0}-\partial_{x} W_{0}\right)+\alpha_{04} W_{1}\left(\partial_{z} U_{0}-\partial_{x} W_{0}\right) \\
& +\alpha_{04} W_{0}\left(\partial_{z} U_{1}-\partial_{x} W_{1}\right)+\alpha_{05} W_{1}\left(\partial_{z} U_{1}-\partial_{x} W_{1}\right) \\
& +\alpha_{11} U_{2} V_{2}+\alpha_{12} W_{2}\left(\partial_{z} U_{2}-\partial_{x} W_{2}\right) \\
& -\alpha_{13} V_{2} \partial_{x} V_{2} \\
& N_{W_{0}}=\alpha_{01} W_{0} V_{1}-\alpha_{06} V_{1} \partial_{z} V_{1}+\alpha_{02} W_{1} V_{1} \\
& +\alpha_{03} U_{0}\left(\partial_{x} W_{0}-\partial_{z} U_{0}\right)+\alpha_{04} U_{1}\left(\partial_{x} W_{0}-\partial_{z} U_{0}\right) \\
& +\alpha_{04} U_{0}\left(\partial_{x} W_{1}-\partial_{z} U_{1}\right)+\alpha_{05} U_{1}\left(\partial_{x} W_{1}-\partial_{z} U_{1}\right) \\
& +\alpha_{11} V_{2} W_{2}-\alpha_{13} V_{2} \partial_{z} V_{2}+\alpha_{12} U_{2}\left(\partial_{x} W_{2}-\partial_{z} U_{2}\right), \\
& N_{U_{1}}=-\alpha_{07} U_{0} V_{1}-\alpha_{08} U_{1} V_{1}+\alpha_{04} W_{0}\left(\partial_{z} U_{0}-\partial_{x} W_{0}\right) \\
& +\alpha_{05} W_{1}\left(\partial_{z} U_{0}-\partial_{x} W_{0}\right)+\alpha_{05} W_{0}\left(\partial_{z} U_{1}-\partial_{x} W_{1}\right) \\
& +\alpha_{09} W_{1}\left(\partial_{x} W_{1}-\partial_{z} U_{1}\right)-\alpha_{14} U_{2} V_{2} \\
& +\alpha_{15} W_{2}\left(\partial_{z} U_{2}-\partial_{x} W_{2}\right)+\alpha_{16} V_{2} \partial_{x} V_{2} \text {, } \\
& N_{W_{1}}=-\alpha_{07} W_{0} V_{1}-\alpha_{08} W_{1} V_{1}+\alpha_{04} U_{0}\left(\partial_{x} W_{0}-\partial_{z} U_{0}\right) \\
& +\alpha_{05} U_{1}\left(\partial_{x} W_{0}-\partial_{z} U_{0}\right)+\alpha_{05} U_{0}\left(\partial_{x} W_{1}-\partial_{z} U_{1}\right) \\
& +\alpha_{09} U_{1}\left(\partial_{z} U_{1}-\partial_{x} W_{1}\right)-\alpha_{14} V_{2} W_{2}+\alpha_{16} V_{2} \partial_{z} V_{2} \\
& +\alpha_{15} U_{2}\left(\partial_{x} W_{2}-\partial_{z} U_{2}\right) \text {, } \\
& N_{V_{1}}=-\alpha_{01} U_{0}^{2}-\alpha_{10} U_{0} U_{1}+a_{08} U_{1}^{2}-\alpha_{01} W_{0}^{2}-a_{10} W_{0} W_{1} \\
& +\alpha_{08} W_{1}^{2}+a_{06} W_{0} \partial_{z} V_{1}+\alpha_{06} U_{0} \partial_{x} V_{1}-\alpha_{17} U_{2}^{2} \\
& -\alpha_{17} W_{2}^{2}+\alpha_{24} W_{2} \partial_{z} V_{2}+\alpha_{24} U_{2} \partial_{x} V_{2} \text {, } \\
& N_{U_{2}}=\alpha_{17} U_{2} V_{1}+\alpha_{18} U_{0} V_{2}+\alpha_{19} U_{1} V_{2} \\
& +\alpha_{12} W_{2}\left(\partial_{z} U_{0}-\partial_{x} W_{0}\right)+\alpha_{15} W_{2}\left(\partial_{z} U_{1}-\partial_{x} W_{1}\right) \\
& +\alpha_{12} W_{0}\left(\partial_{z} U_{2}-\partial_{x} W_{2}\right)+\alpha_{15} W_{1}\left(\partial_{z} U_{2}-\partial_{x} W_{2}\right) \\
& -\alpha_{24}\left(V_{2} \partial_{x} V_{1}+V_{1} \partial_{x} V_{2}\right) \text {, }
\end{aligned}
$$




$$
\begin{aligned}
N_{W_{2}}= & \alpha_{18} V_{2} W_{0}+\alpha_{19} V_{2} W_{1}+\alpha_{17} V_{1} W_{2}-\alpha_{24} V_{2} \partial_{z} V_{1} \\
& -\alpha_{24} V_{1} \partial_{z} V_{2}+\alpha_{12} U_{2}\left(\partial_{x} W_{0}-\partial_{z} U_{0}\right) \\
& +\alpha_{15} U_{2}\left(\partial_{x} W_{1}-\partial_{z} U_{1}\right)+\alpha_{12} U_{0}\left(\partial_{x} W_{2}-\partial_{z} U_{2}\right) \\
& +\alpha_{15} U_{1}\left(\partial_{x} W_{2}-\partial_{z} U_{2}\right), \\
N_{V_{2}}= & -\alpha_{25}\left(U_{0} U_{2}+W_{0} W_{2}\right)-\alpha_{26}\left(U_{1} U_{2}+W_{1} W_{2}\right) \\
& +\alpha_{24}\left(W_{2} \partial_{z} V_{1}+U_{2} \partial_{x} V_{1}\right)+\alpha_{13}\left(W_{0} \partial_{z} V_{2}+U_{0} \partial_{x} V_{2}\right) \\
& -\alpha_{16}\left(W_{1} \partial_{z} V_{2}+U_{1} \partial_{x} V_{2}\right) .
\end{aligned}
$$

The coefficients of the nonlinear terms are

$$
\begin{aligned}
& \alpha_{01}=\sqrt{35 / 11} / 52, \quad \alpha_{02}=45 \sqrt{105 / 11} / 52, \quad \alpha_{03}=21 \sqrt{105} / 286, \\
& \alpha_{04}=\sqrt{35} / 286, \quad \alpha_{05}=19 \sqrt{105 / 286}, \quad \alpha_{06}=\sqrt{105} / 13, \\
& \alpha_{07}=7 \sqrt{105 / 11} / 52, \quad \alpha_{08}=9 \sqrt{35 / 11} / 52, \quad \alpha_{09}=9 \sqrt{35} / 286, \\
& \alpha_{10}=19 \sqrt{105 / 11} / 26, \quad \alpha_{11}=9 \sqrt{35} / 44, \quad \alpha_{12}=5 \sqrt{35 / 3} / 22, \\
& \alpha_{13}=10 \sqrt{105} / 143, \quad \alpha_{14}=7 \sqrt{105} / 44, \quad \alpha_{15}=\sqrt{35} / 22, \\
& \alpha_{16}=15 \sqrt{35} / 143, \quad \alpha_{17}=\sqrt{35 / 11} / 4, \quad \alpha_{18}=\sqrt{35} / 44 \\
& \alpha_{19}=9 \sqrt{105} / 44, \quad \alpha_{24}=5 \sqrt{105 / 11} / 26, \quad \alpha_{25}=5 \sqrt{35} / 22, \\
& \alpha_{26}=\sqrt{105} / 22 .
\end{aligned}
$$

The coefficients of the linear terms are $b_{1}=8 / 11, b_{2}$ $=4 /(11 \sqrt{3}), b_{3}=5 /(11 \sqrt{3}), b_{4}=9 \sqrt{3} / 2$, and $b_{5}=10 / 13$. Furthermore, since we use periodic boundary conditions in the numerical simulation, the mean values of the velocities are computed using the following equations, where the overbar denotes an average in the $(x, z)$ plane:

$$
\begin{aligned}
\left(\frac{d}{d t}+R^{-1} \gamma_{0}\right) \overline{U_{0}}= & -\left(\alpha_{01}-\alpha_{04} \beta_{1}\right) \overline{U_{0} V_{1}} \\
& -\left(\alpha_{02}-\alpha_{05} \beta_{1}\right) \overline{U_{1} V_{1}} \\
& -\left(\alpha_{11}-\alpha_{12} \beta_{2}\right) \overline{U_{2} V_{2}}-b_{4} \overline{U_{1}} / R, \\
\left(\frac{d}{d t}+R^{-1} \gamma_{0}\right) \overline{W_{0}}= & -\left(\alpha_{01}-\alpha_{04} \beta_{1}\right) \overline{W_{0} V_{1}} \\
& -\left(\alpha_{02}-\alpha_{05} \beta_{1}\right) \overline{W_{1} V_{1}} \\
& -\left(\alpha_{11}-\alpha_{12} \beta_{2}\right) \overline{W_{2} V_{2}}-b_{4} \overline{W_{1}} / R, \\
\left(\frac{d}{d t}+R^{-1} \gamma_{1}\right) \overline{U_{1}}= & \left(\alpha_{07}+\beta_{1} \alpha_{05}\right) \overline{U_{0} V_{1}} \\
& -\left(\alpha_{09} \beta_{1}-\alpha_{08}\right) \overline{U_{1} V_{1}} \\
& +\left(\alpha_{14}+\alpha_{15} \beta_{2}\right) \overline{U_{2} V_{2}}-b_{4} \overline{U_{0}} / R,
\end{aligned}
$$

$$
\begin{aligned}
\left(\frac{d}{d t}+R^{-1} \gamma_{1}\right) \overline{W_{1}}= & \left(\alpha_{07}+\beta_{1} \alpha_{05}\right) \overline{W_{0} V_{1}} \\
& -\left(\alpha_{09} \beta_{1}-\alpha_{08}\right) \overline{W_{1} V_{1}} \\
& +\left(\alpha_{14}+\alpha_{15} \beta_{2}\right) \overline{W_{2} V_{2}}-b_{4} \overline{W_{0}} / R, \\
\left(\frac{d}{d t}+R^{-1} \gamma_{2}\right) \overline{U_{2}}= & \left(\alpha_{15} \beta_{2}-\alpha_{17}\right) \overline{U_{2} V_{1}} \\
& +\left(\alpha_{15} \beta_{2}-\alpha_{19}\right) \overline{U_{1} V_{2}} \\
& +\left(\alpha_{12} \beta_{2}-\alpha_{18}\right) \overline{U_{0} V_{2}}
\end{aligned}
$$

$$
\begin{aligned}
\left(\frac{d}{d t}+R^{-1} \gamma_{2}\right) \overline{W_{2}}= & \left(\alpha_{15} \beta_{2}-\alpha_{17}\right) \overline{W_{2} V_{1}} \\
& +\left(\alpha_{15} \beta_{2}-\alpha_{19}\right) \overline{W_{1} V_{2}} \\
& +\left(\alpha_{12} \beta_{2}-\alpha_{18}\right) \overline{W_{0} V_{2}} .
\end{aligned}
$$

${ }^{1}$ D. R. Carlson, S. E. Widnall, and M. F. Peeters, "A flow-visualization study of transition in plane Poiseuille flow," J. Fluid Mech. 21, 487 (1982).

${ }^{2}$ F. Alavyoon, D. S. Henningson, and P. H. Alfredsson, "Turbulent spots in plane Poiseuille flow-flow visualization," Phys. Fluids 29, 1328 (1986).

${ }^{3}$ O. Dauchot and F. Daviaud, "Finite amplitude perturbation and spots growth-mechanism in plane Couette flow," Phys. Fluids 7, 335 (1995).

${ }^{4}$ M. Gad-El-Hak, R. F. Blackwelder, and J. J. Riley, "On the growth of turbulent regions in laminar boundary layers," J. Fluid Mech. 110, 73 (1981).

${ }^{5}$ B. G. B. Klingmann and P. H. Alfredsson, "Turbulent spots in plane Poiseuille flow-measurements of the velocity-field," Phys. Fluids A 2, 2183 (1990).

${ }^{6}$ A. Lundbladh and A. V. Johansson, "Direct simulation of turbulent spots in plane Couette flow," J. Fluid Mech. 229, 499 (1991).

${ }^{7}$ J. Schumacher and B. Eckhardt, "Evolution of turbulent spots in a parallel shear flow," Phys. Rev. E 63, 046307 (2001).

${ }^{8} \mathrm{P}$. Manneville, "Spots and turbulent domains in a model of transitional plane Couette flow," Theor. Comput. Fluid Dyn. 18, 169 (2004).

${ }^{9}$ J. Mathew and A. Das, "Direct numerical simulations of spots," Curr. Sci. 79, 816 (2000).

${ }^{10}$ R. L. Panton, "Overview of the self-sustaining mechanisms of wall turbulence," Prog. Aerosp. Sci. 37, 341 (2001).

${ }^{11} \mathrm{~F}$. Li and S. E. Widnall, "Wave patterns in plane Poiseuille flow created by concentrated disturbances," J. Fluid Mech. 208, 639 (1989).

${ }^{12}$ D. S. Henningson, P. Spalart, and J. Kim, "Numerical simulations of turbulent spots in plane Poiseuille and boundary-layer flow," Phys. Fluids 30, 2914 (1987).

${ }^{13}$ D. S. Henningson, "Wave growth and spreading of a turbulent spot in plane Poiseuille flow," Phys. Fluids A 1, 1876 (1989).

${ }^{14}$ D. S. Henningson and P. H. Alfredsson, "The wave structure of turbulent spots in plane Poiseuille flow," J. Fluid Mech. 178, 405 (1987).

${ }^{15}$ M. Lagha and P. Manneville, "Modeling transitional plane Couette flow," Eur. Phys. J. B 58, 433 (2007).

${ }^{16} \mathrm{M}$. Lagha and P. Manneville, "Modeling of plane Couette flow. I. Large scale flow around turbulent spots," Phys. Fluids 19, 094105 (2007).

${ }^{17}$ M. Lagha, "Modeling of plane Couette flow. II. On the spreading of a turbulent spot," Phys. Fluids 19, 104108 (2007).

${ }^{18}$ D. S. Henningson and J. Kim, "On turbulent spots in plane Poiseuille flow," J. Fluid Mech. 228, 183 (1991).

${ }^{19}$ The evolution of the same spot is further illustrated in accompanying video files showing $U_{0}, U_{2}, V_{2}$, and $W_{2}$.

${ }^{20}$ D. V. Papavassiliou and T. J. Hanratty, "Interpretation of large-scale structures observed in a turbulent plane Couette flow," Int. J. Heat Fluid Flow 18, 55 (1997). 